\title{
.
}

\section{RedIRIS, RED ESPAÑOLA DE COMUNICACIONES AVANZADAS PARA EL SECTOR ACADÉMICO Y CIENTÍFICO, Y SU CONTRIBUCIÓN AL DESARROLLO TECNOLÓGICO Y ECONÓMICO}

Todos los países desarrollados han apostado por el modelo de Redes Académicas y de Investigación Nacionales (National Research and Education Networks, NREN), en el que encargan a un organismo proveer de servicios de conectividad avanzada específicos para los centros universitarios y científicos, junto con otros servicios TIC centralizados. En este artículo se explica el contexto político y económico en que se enmarca este modelo, se explican sus características principales y sus ventajas, y se expone la situación en España, en la que RedIRIS actúa como NREN española desde 1988. Se explica su evolución, sus principales líneas estratégicas y el valor añadido que aporta, que puede ser extrapolado a otras áreas.

Palabras clave: conectividad, servicios TIC compartidos, e-ciencia, ICTS, universidades, investigación. Clasificación JEL: L86, O38.

\section{Contexto político y económico de las redes académicas y de investigación}

Desde los años ochenta, los Gobiernos de los países tecnológicamente más avanzados procedieron a crear organismos encargados de proporcionar servicios avanzados de conectividad a los sectores académicos y científicos nacionales, dando lugar a la aparición de las NREN (National Research and Education

* Doctor en Derecho. Director de RedIRIS y de Dominios.es, entidad pública empresarial Red.es

Versión de febrero de 2020.

DOI: https://doi.org/10.32796/bice.2020.3120.6975
Networks, Redes Académicas y de Investigación Nacionales) ${ }^{1}$. Esas redes fueron pioneras en la introducción de internet en sus respectivos países, y han ido evolucionando con la tecnología, de manera que hoy muchas NREN pueden ofrecer a sus usuarios múltiples conexiones de $100 \mathrm{Gbps}$ (100.000 Mbps), frente a los $600 \mathrm{Mbps}$ de las ofertas convencionales de fibra óptica para el sector residencial.

Los Gobiernos financian las NREN para, entre otras cosas, favorecer la colaboración y el intercambio de información entre los centros educativos y científicos a escala nacional y $\triangleright$

1 Davies y Bressan (2010), y en España, el capítulo dedicado a las redes académicas y de investigación en Cómo creamos Internet (Vèa 2013). 
global, y para impulsar la experimentación de internet de nueva generación (la NREN norteamericana se denomina «Internet2»).

Antes de analizar la problemática concreta de las NREN hay que situar esas redes académicas y científicas dentro del marco político, económico, social y tecnológico que justifica la acción del Estado en esta materia y que explica su carácter estratégico.

\subsection{La sociedad de la información y la economía del conocimiento}

El desarrollo de internet y de las comunicaciones electrónicas (las tecnologías de la información y la comunicación, TIC) está influyendo de forma decisiva en la forma en que las personas se comunican, se informan, compran y venden, o establecen sistemas complejos de producción con intercambios de información en tiempo real a escala global. Estos avances han hecho que se acuñe la expresión «sociedad de la información» para definir aquellas sociedades en la que, gracias a las TIC, la creación, distribución, uso, integración y manipulación de la información juega un papel fundamental en la esfera política, económica y cultural. La consecuencia económica de la implantación de una sociedad de la información es el desarrollo de un modelo productivo basado en la economía del conocimiento.

El conocimiento siempre ha sido relevante para la actividad económica, pero, en el actual entorno globalizado, desempeña un papel cada vez más importante, ya que las actividades más cualificadas y que requieren mayor formación y mayor manejo de información son las que generan más valor añadido y las menos susceptibles de deslocalización.

La economía del conocimiento se caracteriza por utilizar el conocimiento como elemento fundamental para generar valor en los productos y servicios en cuyo proceso de creación o transformación participa. El conocimiento es más que mera información. La información son datos procesados con una utilidad general, mientras que el conocimiento significa formas, métodos y maneras de abordar y resolver problemas; significa, entre otras muchas cosas, know how, know who o herramientas o medios de producción para producir o más conocimiento, o productos y servicios con un valor añadido, útil y cuantificable para la sociedad.

Gracias a las TIC han aparecido nuevas industrias (software, comunicaciones electrónicas y aplicaciones) basadas en el conocimiento y se han rejuvenecido las industrias tradicionales. La llamada globalización económica ha sido posible, entre otras cosas, gracias a logros tecnológicos, que han permitido una nueva división del trabajo basada en las cadenas globales de producción, en las que los elementos estratégicos, de mayor valor añadido y de mayor estabilidad, quedan en manos de los países que han centrado su desarrollo en ese modelo de economía del conocimiento, frente a sistemas económicos menos avanzados, basados en la venta de materias primas (cuyas demanda y precios sufren a menudo grandes fluctuaciones), y/o en manufacturas de poca especialización, que aportan poco valor añadido y por las que compiten, gracias a la disminución de los costes de transporte, muchos países en todo el planeta.

\subsection{La ciencia y la investigación en la economía del conocimiento: la e-ciencia}

En el plano político-económico, los países desarrollados se han orientado a la inversión $\triangleright$ 
en investigación y desarrollo tecnológico, en educación, y en el impulso de sistemas nacionales de innovación, como elementos fundamentales para el desarrollo del país.

La acción pública de fomento del I+D+i se plasma en planes de apoyo al desarrollo de infraestructuras científicas y a proyectos de investigación en ciertos sectores clave.

En los distintos planes de fomento de la investigación y la ciencia, tanto europeos como nacionales, una de las áreas más destacadas y a las que más recursos se dedican es a las TIC. Se busca con ello tanto desarrollar nuevos protocolos y servicios que incrementen el rendimiento y la eficiencia de las TIC como aumentar su penetración y uso en todos los segmentos de la sociedad, incluyendo su uso avanzado por la comunidad académica y científica.
Se espera de esa comunidad académica y científica que mejore de forma significativa la formación y la innovación, como elementos claves de la competitividad en una economía del conocimiento. Para lograr ese resultado, la comunidad académica y científica debe hacer un uso adecuado de las TIC, que aumentarán su productividad y su capacidad de interactuar con aquellos grupos que más valor puedan aportarles, con independencia de su ubicación.

Si bien todos los grupos de docentes e investigadores deben usar en mayor o menor medida las TIC, existe una serie de colectivos que hacen un uso especialmente intenso de ellas, por la necesidad de realizar simulaciones telemáticas o cálculos complejos o de cooperar de forma remota con otros grupos.

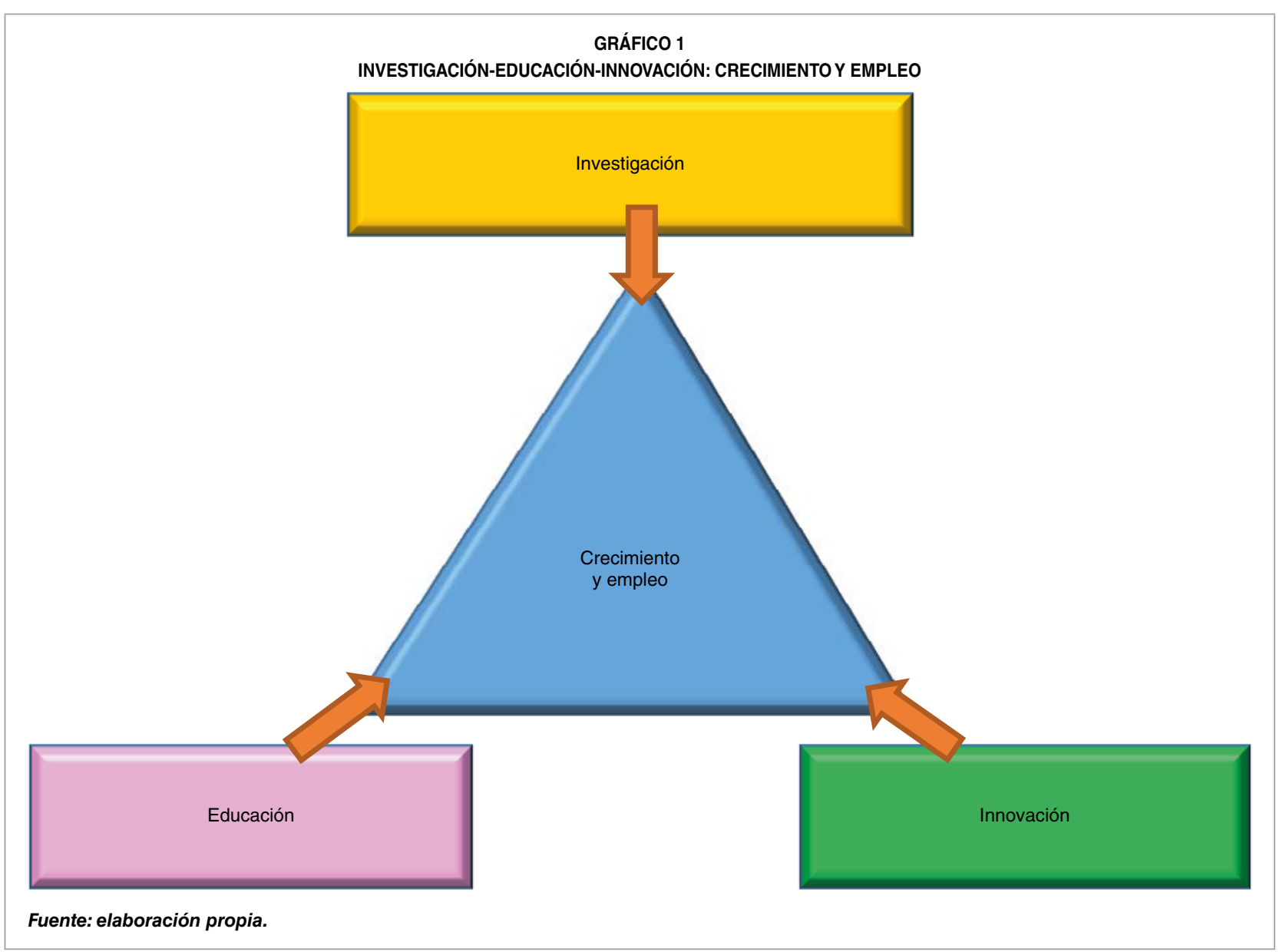


En este contexto, se habla de e-ciencia para referirse al conjunto de actividades científicas desarrolladas mediante el uso de recursos distribuidos accesibles a través de internet. Hoy en día, cálculo, almacenamiento y acceso a la información, entre otros, constituyen los principales servicios utilizados y compartidos mediante redes de comunicaciones de alta velocidad dedicadas a la investigación, cuyo desarrollo, junto al de las tecnologías y aplicaciones colaborativas, están creando un escenario idóneo para la interacción entre investigadores.

En El libro blanco de la e-ciencia en España (FECYT, 2007) se establecía una taxonomía de la e-ciencia, en la que se diferenciaban, por un lado, unas líneas horizontales de infraestructuras y servicios comunes y, por otro lado, unas líneas verticales de principales grupos de usuarios de las líneas horizontales.
En relación con las líneas verticales, como ya se ha indicado, todos los miembros de la comunidad académica y científica tendrán que usar las TIC, pero hay una serie de ámbitos en los que, por el tipo de investigación a realizar, este uso de las TIC es especialmente intenso.

En ese sentido, destaca, por ejemplo, la física de altas energías. Sus necesidades TIC son tan elevadas que algunas de las primeras redes de comunicación científicas se establecieron para interconectar centros de física de altas energías (que, hoy, siguen encontrándose entre los principales usuarios de las redes académicas y de investigación). Otras áreas que hacen uso especialmente intenso de las TIC son la Astronomía, la Biomedicina, la Ciencia de Materiales, las Ciencias de la Tierra, la Física, la Química, la Ingeniería y la propia investigación en nuevos desarrollos TIC.

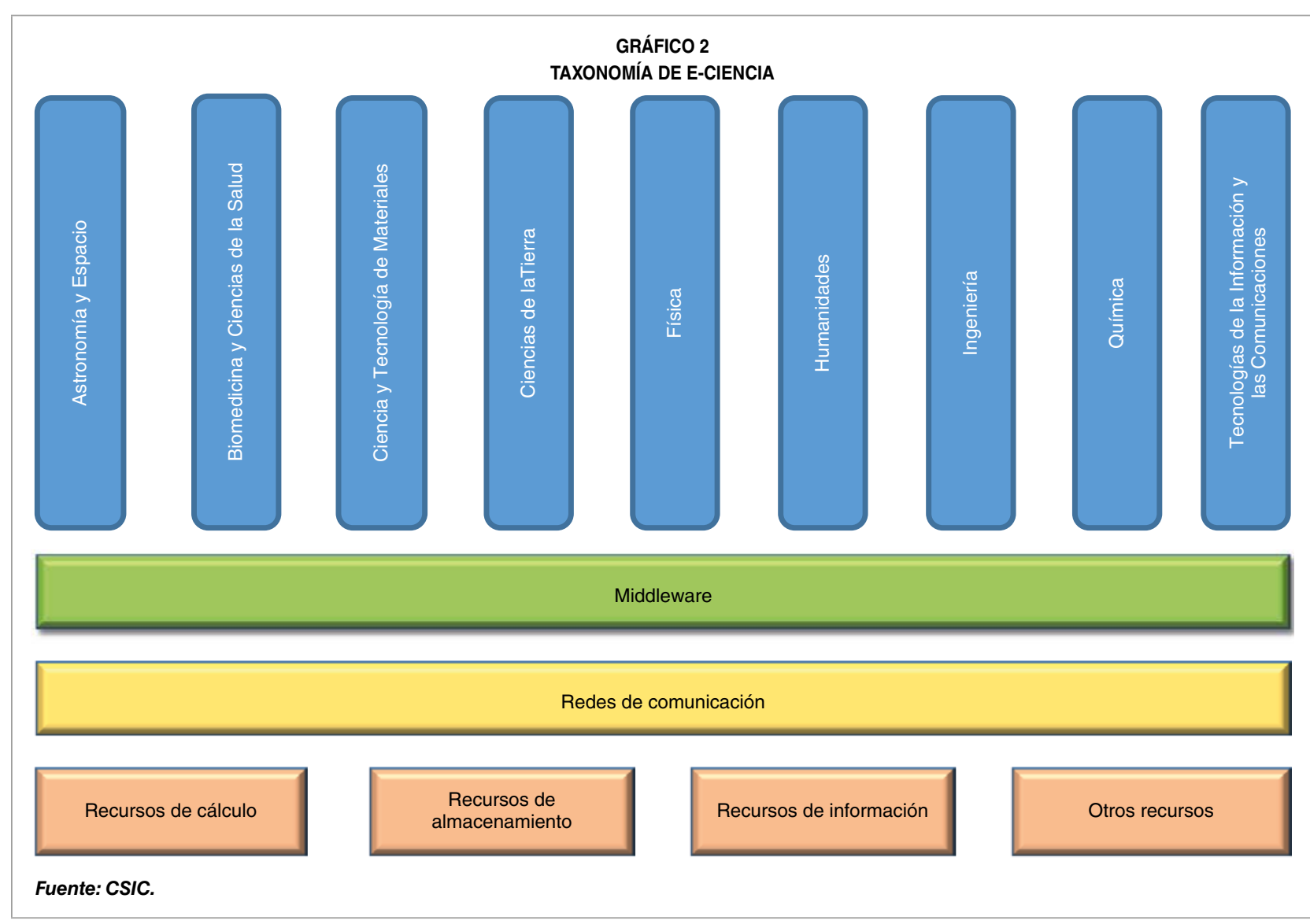


Por ejemplo, hay especialistas de biotecnología que pueden realizar in silico, es decir, en ordenadores, experimentos simulados que es muy costoso realizar in vitro, y que podrán avanzar más que aquellos de sus colegas que no usan esos medios. O hay especialistas en meteorología que pueden realizar complejas simulaciones que, gracias a los supercomputadores, se ejecutan en unos pocos minutos, pudiendo lanzar rápidamente nuevas simulaciones con hipótesis más ajustadas. Un meteorólogo que ejecutase esos mismos cálculos sobre un cluster local de ordenadores de su centro tendrá mucha menos capacidad de procesamiento de datos y tendrá un rendimiento inferior (tardará horas o días en realizar el mismo cálculo).

Los científicos también se benefician cada vez en mayor medida de las posibilidades del big data y de la inteligencia artificial, y disponen de acceso bajo demanda a los recursos que necesitan gracias a servicios cloud, que se adaptan mejor a cargas de trabajo variables, permiten acceso compartido a los recursos y disponen de servicios de soporte centralizados.

En ese sentido, hay que destacar iniciativas como EOSC (European Open Science Cloud) ${ }^{2}$,

$2 \operatorname{COM}(2016) 178$ final. que tienen como objetivo que todos los datos generados por la investigación realizada con financiación pública sean abiertos, y que además estén en la nube y que cumplan con los principios FAIR (Findable, Accesible, Interoperable, Reusable; o Encontrables, Accesibles, Interoperables y Reutilizables). Esto debería permitir ejecutar algoritmos de big data e inteligencia artificial sobre conjuntos de datos mucho mayores $^{3}$, y multisectoriales, así como posibilitar que terceros pudieran verificar la replicabilidad de las conclusiones de ciertas investigaciones.

Los científicos activos en el ámbito de e-ciencia desarrollan aplicaciones específicas para sus necesidades científicas, que luego pueden ejecutar gracias a elementos horizontales de e-ciencia (recursos de computación, de almacenamiento, redes académicas...) para utilizarlas. Este es uno de los principales problemas para el desarrollo de la e-ciencia: hay científicos que trabajan coordinadamente con informáticos para definir las aplicaciones que necesitan, mientras que otros no lo hacen.

Esto hace que, por un lado, haya científicos, activos en e-ciencia, con las aplicaciones $\triangleright$

3 https://www.economist.com/leaders/2017/05/06/the-worlds-most valuable-resource-is-no-longer-oil-but-data

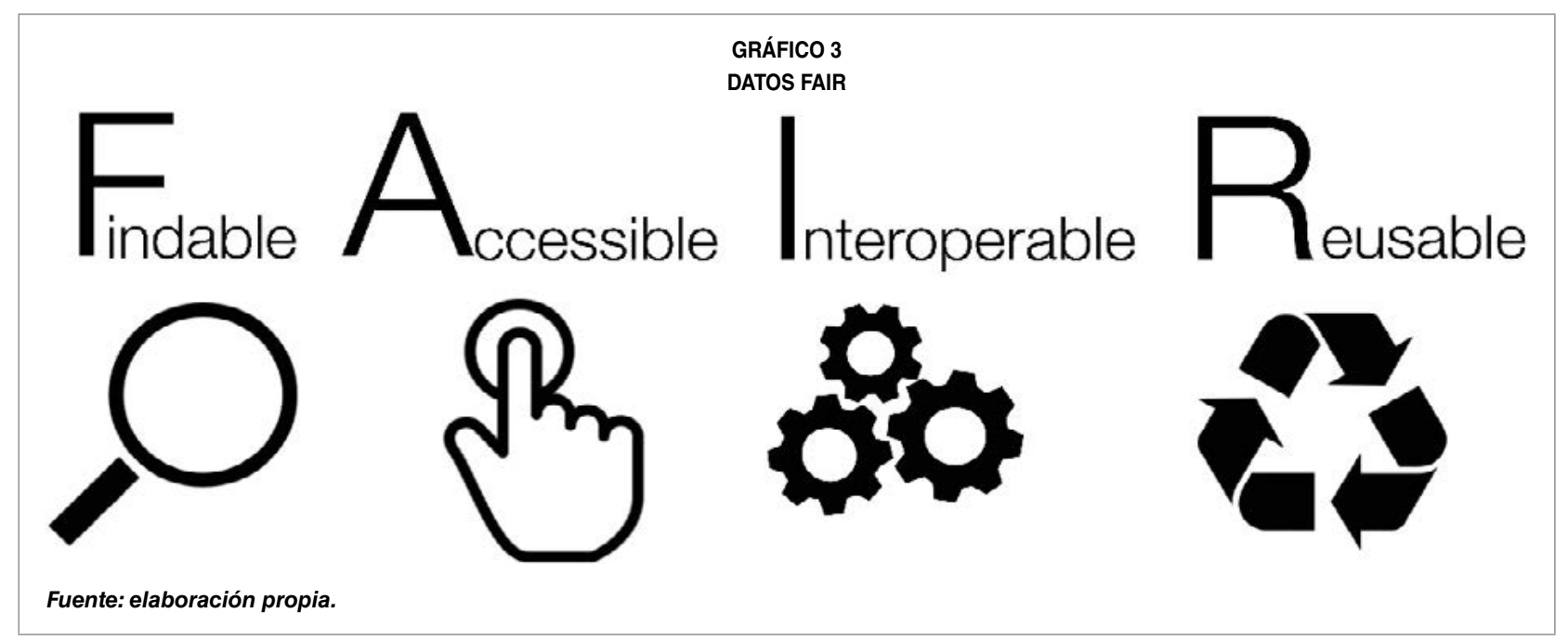


que necesitan, y que colaboran de forma remota con los grupos referentes en su ámbito, consiguiendo un rendimiento muy alto; y otros científicos que no usan esos recursos y que están en una posición poco competitiva.

En ese sentido, los sistemas de ciencia no solo necesitan incrementar los fondos para I+D, sino velar por su eficacia, evaluando adecuadamente qué grupos están usando metodologías y recursos que les permiten ofrecer valor, poner datos de interés a disposición de terceros y desarrollar ciencia de frontera; y qué grupos no. En ciertas disciplinas científicas es condición necesaria (aunque no suficiente) emplear ciertos recursos TIC, incluyendo una conexión de muy alta capacidad que sea fiable y segura.

Algo similar sucede en el ámbito académico, con la importancia creciente del aprendizaje online, en un ciclo formativo constante (que se extiende más allá del periodo de estudios escolares y universitarios) y en el que las nuevas tecnologías permiten, por ejemplo, seguir en directo desde cualquier parte del planeta lecciones magistrales de los docentes más destacados.

\section{Las Redes Académicas y de Investigación Nacionales (NREN)}

\subsection{Función y ventajas de las NREN}

Las NREN cumplen una serie de funciones de interés general ${ }^{4}$, de gran importancia sobre todo para la e-ciencia:

- Son redes de datos que permiten la compartición de recursos comunes, sobre

4 Sobre las ventajas de las Redes Académicas y de Investigación Nacionales (NREN), ver https://www.casefornrens.org/ todo en el nuevo paradigma de la ciencia abierta en la nube.

- Hacen posible la cooperación de investigadores y personal académico con aquellos que más valor pueden aportar a su trabajo, con independencia de dónde se encuentren localizados.

- Ayudan a luchar contra la brecha digital, evitando la necesidad de emigrar y la consiguiente «fuga de cerebros».

- Son bancos de pruebas para el desarrollo de nuevos protocolos y servicios TIC.

- Permiten optimizar el despliegue de e-infraestructuras, ya que facilita el acceso remoto a las mismas, evitando la necesidad de desplegar pequeñas e-infraestructuras locales, menos potentes y más caras de mantener.

- Son infraestructuras que favorecen el impulso de la sociedad de la información ${ }^{5}$.

Todas esas ventajas se dan si la red académica tiene capacidad de transmisión suficiente, y si además no hay cuellos de botella, y se dispone de esa capacidad en todos los niveles de la Intranet Global de la Investigación (red de campus, red regional, red nacional y redes internacionales).

Los gestores de las redes académicas trabajan para que el investigador, que normalmente será un especialista en su materia (física, biomedicina, ciencias de la tierra, etcétera), pero no en tecnologías TIC, acceda al $D$

\footnotetext{
5 Las NREN ofrecen capacidades superiores a las de las ofertas comerciales; incorporan protocolos que no se usan (o no de forma generalizada) en redes comerciales, como era el caso de IPv6 o Multicast; y fomentan despliegue de nuevas infraestructuras, como un segundo cable a Canarias a raíz de la adjudicación del concurso de RedIRIS-NOVA en 2009 (ver Informe de competencia en el mercado de la banda ancha en Canarias, Observatorio Canario de Telecomunicaciones y para la Sociedad de la Información, 2011, https://www.octsi.es/images/documentos/informe banda ancha_2011.pdf); o un cable directo entre Latinoamérica y Europa a raíz del proyecto BELLA (actualmente en ejecución); ver https://ec.europa.eu/digital-single-market/en/news/bella-new-digitaldata-highway-between-europe-and-latin-america
} 


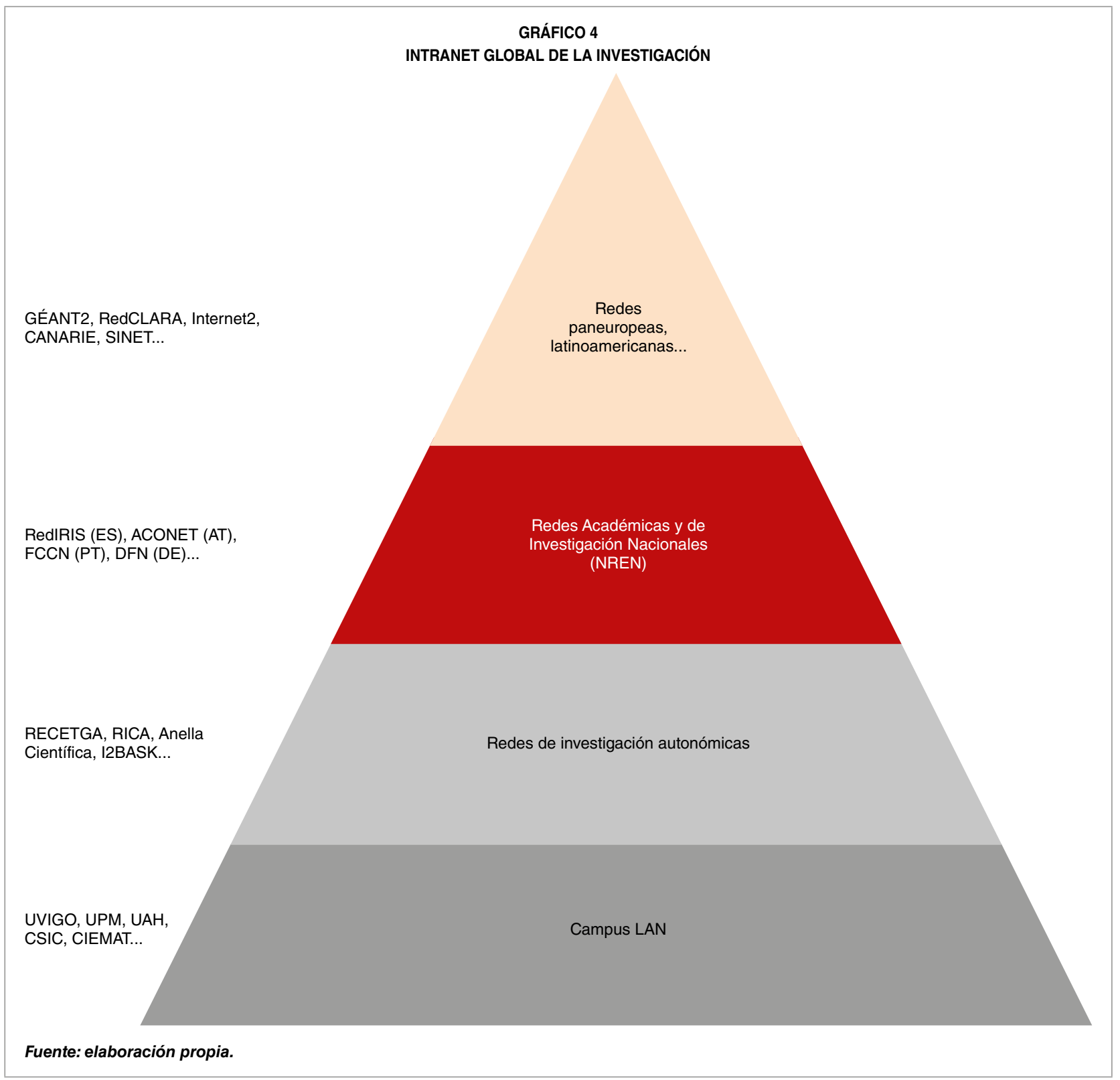

servicio de forma sencilla, transparente, ágil, estable, segura y económica.

Sobre estas premisas genéricas, comunes a todas las redes académicas, cada una de ellas tiene elementos específicos, en la forma de organización y financiación, en los usuarios a los que da servicio, en los servicios que ofrece...

Las redes académicas actúan en todo caso como centrales de compra de conectividad a proveedores privados (obteniendo importantes economías de escala y de enfoque), y son además asesores de las autoridades públicas que financian esos servicios, ya que permiten a los Gobiernos disponer de un organismo que:

- Que está al tanto de la tecnología disponible.

- Que puede redactar los pliegos por los que se contrata, de forma centralizada, los servicios de conectividad avanzada que demanda la comunidad académica y de investigación. 
- Que puede evaluar las ofertas, y luego verificar el cumplimiento de los SLA (Service Level Agreements, niveles de servicio establecidos y aceptados por el proveedor, que suele ser penalizado si incumple esos SLA).

Las redes académicas y de investigación son además percibidas por sus usuarios como independientes de intereses comerciales y como valiosos asesores en temas relacionados con conectividad, con seguridad y con ciertos servicios TIC, como identidad digital, herramientas colaborativas o servicios cloud (cuya prestación no es posible sin servicios de conectividad adecuados).

Las redes académicas y de investigación no solo agrupan la demanda de sus usuarios, $D$

GRÁFICO 5

TOPOLOGÍA DE LA RED ACADÉMICA Y CIENTÍFICA PANEUROPEA GÉANT

\section{GÊANT}

La red para empresas académicas y científicas, GÉANT, interconecta a las Redes Académicas y de Investigación Nacionales (NREN). En conjunto, conectar unos 50 millones de usuarios y 10.000 instituciones a lo largo de Europa.

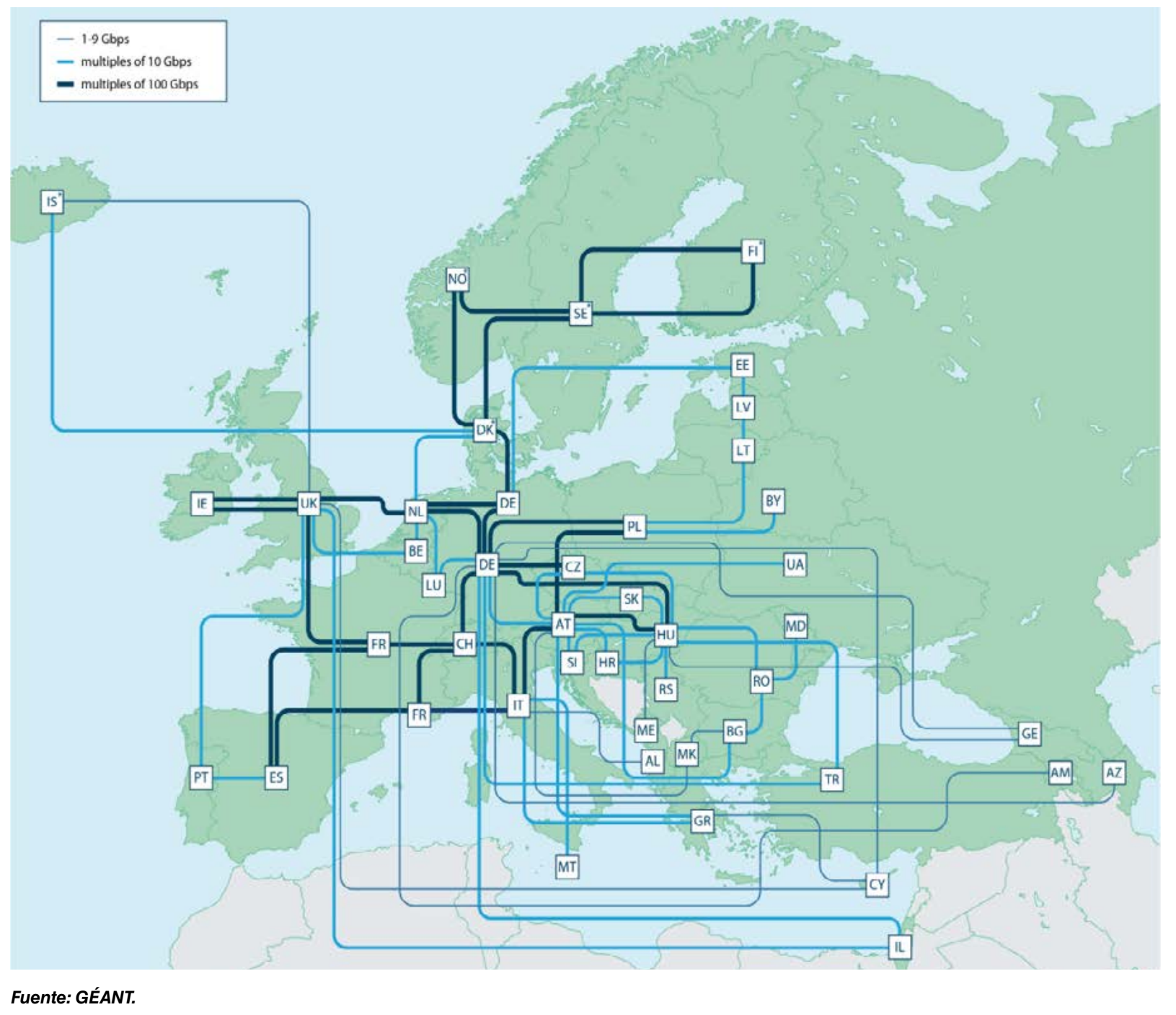


sino que establecen condiciones de servicio que estos difícilmente podrían darse por sí solos. Por ejemplo, las redes académicas buscan garantizar la estabilidad del servicio, a través del diseño de infraestructuras malladas que garantizan una redundancia que los usuarios difícilmente obtendrían desplegando cada uno su servicio. También llegan a acuerdos con otras redes académicas autonómicas e internacionales para garantizar la mejor conectividad posible, con control de extremo a extremo, y con servicios tecnológicamente avanzados (en ocasiones no disponibles comercialmente) e interoperables, lo que no podrían hacer centros científicos actuando de forma aislada.

Destaca la importancia del papel de coordinación que desempeñan:

- A escala nacional, las NREN, en estrecha cooperación con redes académicas y científicas autonómicas y con redes de campus.
- A escala internacional, las asociaciones continentales de NREN, como GÉANT en Europa, que:

- Contrata servicios comunes para todos sus miembros (como la red académica paneuropea, y sus conexiones transatlánticas; los certificados digitales; o su acuerdo marco para servicios cloud, con una sola licitación para 40 NREN, y para las 10.000 instituciones académicas y científicas afiliadas a esas redes académicas ${ }^{6}$ ).

- Desarrolla servicios compartidos, como el sistema de movilidad inalámbrica eduroam, que permite a los miembros de la comunidad académica $y$ científica acceder, de forma sencilla $\triangleright$

6 Ver «Game-changer for cloud computing: GÉANT ensures easy consumption of laaS cloud services across European research and education community", GÉANT News: https://www.geant.org/News_and_ Events/Pages/Game-changer-for-cloud-computing.aspx

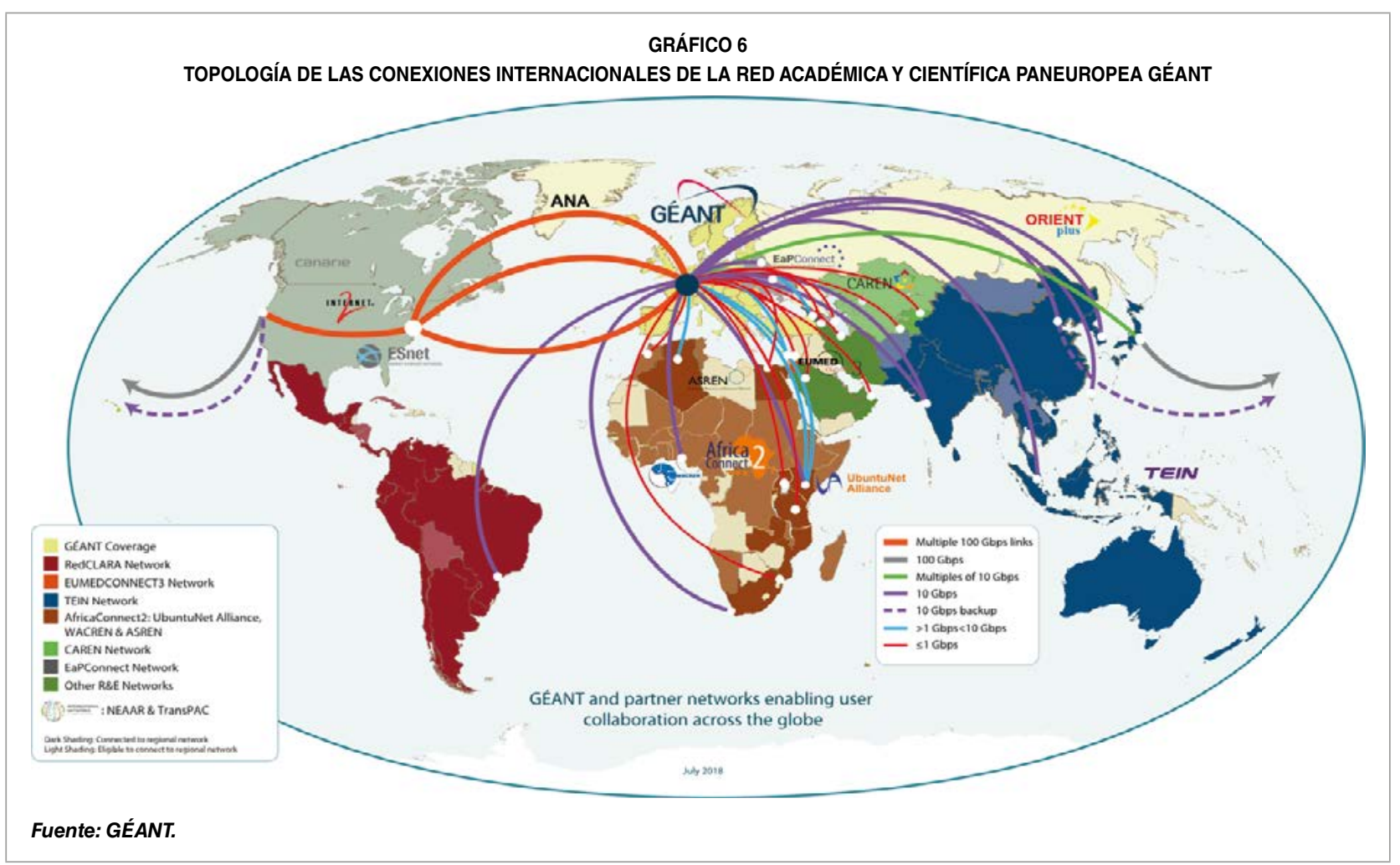


y segura, a miles de redes inalámbricas de universidades y centros de investigación en más de cien países, a través de un sistema federado de identidad digital en el que usan las mismas claves de acceso habituales de su centro de origen.

\subsection{Modelos de gestión de las NREN}

Todos los países más desarrollados económicamente disponen de Redes Académicas y de Investigación Nacionales, pero en cada uno de ellos se han organizado con modelos propios, en función de las misiones encomendadas a esas redes, de la tradición jurídica y organizativa del país, de su estructura territorial, etcétera.

Entre los aspectos más relevantes de los distintos modelos se encuentran los siguientes.

\subsubsection{Modelo organizativo de las redes académicas y de investigación}

Las Redes Académicas y de Investigación Nacionales son financiadas, de forma directa o indirecta, por los Gobiernos, pero la gestión pueden asumirla directamente estos, o confiarla a algún organismo sin ánimo de lucro (p. ej., una fundación), según se quiera fomentar una mayor dependencia o independencia respecto al Gobierno a la hora de organizar el servicio y de permitir que participen en su gestión las instituciones afiliadas.

\subsubsection{Sistemas de financiación de las redes académicas y de investigación}

Muchas Redes Académicas y de Investigación Nacionales tienen un coste significativo en términos absolutos (más de diez redes académicas europeas tienen un presupuesto $D$

\begin{tabular}{|c|} 
GRÁFICO 7 \\
MAPA DE EDUROAM \\
\hline ¿Dónde puedo hacer eduroam? \\
\hline $\begin{array}{c}\text { Iniciado en Europa, eduroam (contracción de educational roaming) se ha expandido rápidamente } \\
\text { en la comunidad científica y académica y está disponible en } 101 \text { países. }\end{array}$ \\
\hline
\end{tabular}

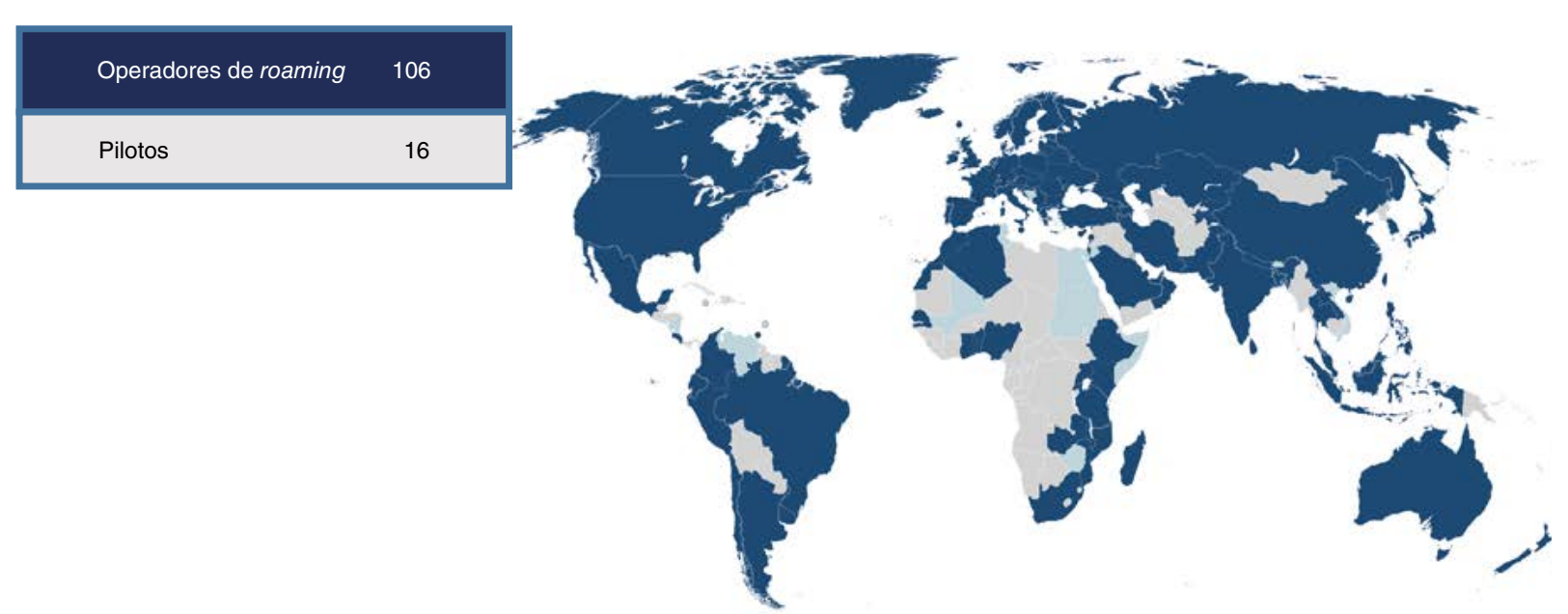

Fuente: Eduroam 


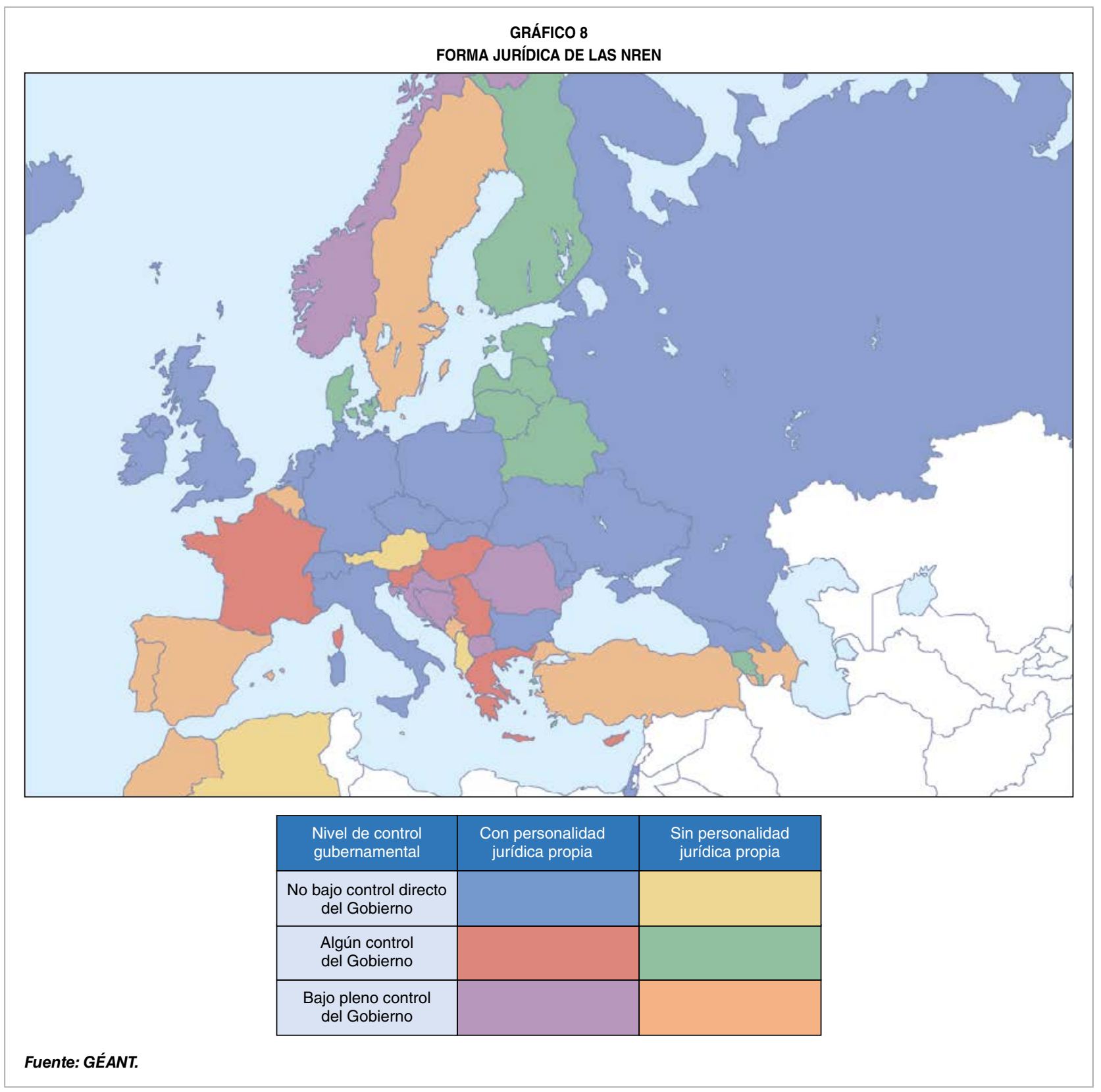

superior a 10 millones de euros/año), aunque en términos relativos suponen un importante ahorro frente a modelos alternativos?.

Algunas redes académicas reciben toda 0 casi toda su financiación directamente del Gobierno nacional, mientras que otras reciben la

7 Ver, por ejemplo, «NREN Network Access - Evaluating Value for money».https://www.casefornrens.org/Resources_and_Tools/Document_ Library/Documents/NREN\%20network\%20access $\% 20 \%$ E2\%80\%90\%20 evaluating \%20value\%20for\%20money.pdf mayoría de sus fondos de sus usuarios (universidades y centros científicos, que reciben a su vez el dinero del Estado).

Los modelos en los que las NREN reciben los fondos de sus usuarios obligan a las redes académicas a justificar mejor ante estos sus servicios, pero hay que establecer complejos sistemas de reparto de costes entre las instituciones afiliadas, y puede haber problemas para financiar servicios innovadores. 


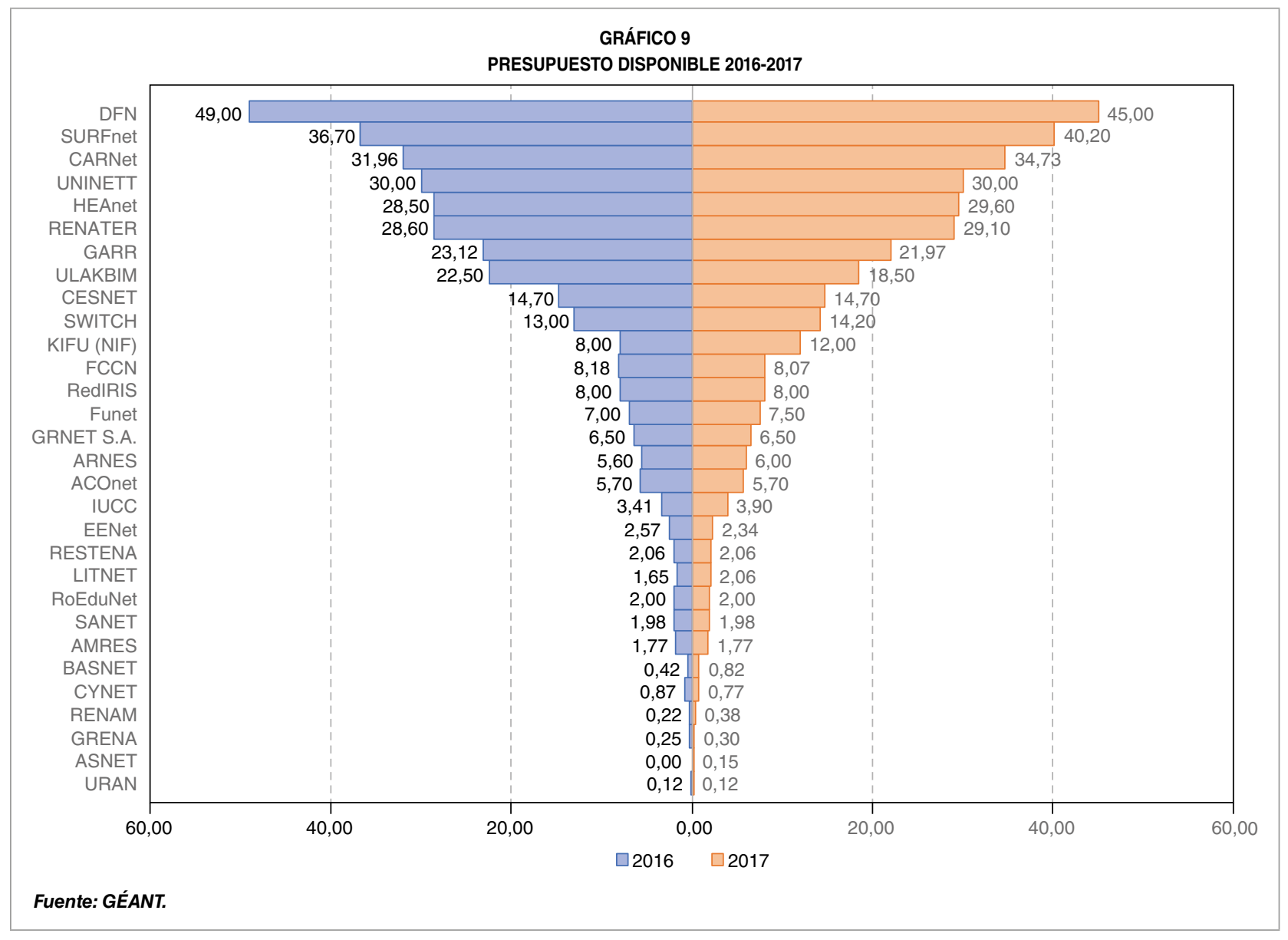

El modelo centralizado facilita el despliegue de servicios avanzados y simplifica el proceso de gestión y de toma de decisión, a costa de perder relación con las instituciones afiliadas y de tener una gran dependencia del importe de la partida presupuestaria correspondiente.

Se considera normalmente que, para los aspectos más relacionados con innovación o con reducción de la brecha digital, puede ser conveniente, en todo caso, que haya al menos un cierto grado de financiación centralizada.

\subsubsection{Políticas de acceso y uso de las redes académicas}

Las Redes Académicas y de Investigación Nacionales están concebidas como redes privadas para grupos cerrados de usuarios, definidos en sus políticas de afiliación, que además tienen establecidas políticas de uso aceptable, que determinan qué uso legítimo pueden hacer de las NREN sus usuarios.

Estas premisas son importantes a la hora de establecer que las redes académicas no son operadores de redes o servicios de comunicaciones electrónicas disponibles al público, y que no están compitiendo con las empresas del sector para captar clientes privados, sino que se están sirviendo de esas empresas para conectar entre sí a organismos de su sistema académico o de $\mathrm{I}+\mathrm{D}+\mathrm{i}$.

Dentro de ese marco, cada red académica y de investigación determina qué colectivos de usuarios van a poder hacer uso de sus servicios. 
RedIRIS, RED ESPAÑOLA DE COMUNICACIONES AVANZADAS...

CUADRO 1

FUENTE DE INGRESOS ESTIMADOS POR NREN-2017

\begin{tabular}{|c|c|c|c|c|c|c|}
\hline & $\begin{array}{l}\text { Instituciones } \\
\text { clientes }\end{array}$ & Comerciales & $\begin{array}{l}\text { Subvencion de } \\
\text { GÉANT }\end{array}$ & $\begin{array}{l}\text { Gobierno o } \\
\text { entidades } \\
\text { públicas }\end{array}$ & Otras UE & Otras \\
\hline \multicolumn{7}{|l|}{ ACOnet } \\
\hline \multicolumn{7}{|l|}{ AMRES } \\
\hline \multicolumn{7}{|l|}{ ARNES } \\
\hline \multicolumn{7}{|c|}{ ASNET-AM } \\
\hline \multicolumn{7}{|c|}{ AzScienceNet } \\
\hline \multicolumn{7}{|l|}{ BASNET } \\
\hline \multicolumn{7}{|l|}{ CARNet } \\
\hline \multicolumn{7}{|l|}{ CESNET } \\
\hline \multicolumn{7}{|l|}{ CYNET } \\
\hline \multicolumn{7}{|l|}{ DFN } \\
\hline \multicolumn{7}{|l|}{ EENet } \\
\hline \multicolumn{7}{|l|}{ FCCN } \\
\hline \multicolumn{7}{|l|}{ Funet } \\
\hline \multicolumn{7}{|l|}{ GARR } \\
\hline \multicolumn{7}{|l|}{ GRENA } \\
\hline \multicolumn{7}{|c|}{ GRNET SA } \\
\hline \multicolumn{7}{|l|}{ HEAnet } \\
\hline \multicolumn{7}{|l|}{ IUCC } \\
\hline \multicolumn{7}{|c|}{ KIFU (NIIF) } \\
\hline \multicolumn{7}{|l|}{ LITNET } \\
\hline \multicolumn{7}{|l|}{ MREN } \\
\hline \multicolumn{7}{|l|}{ RedIRIS } \\
\hline \multicolumn{7}{|l|}{ RENAM } \\
\hline \multicolumn{7}{|l|}{ RENATER } \\
\hline \multicolumn{7}{|l|}{ RESTENA } \\
\hline \multicolumn{7}{|l|}{ RoEduNet } \\
\hline \multicolumn{7}{|l|}{ SUNET } \\
\hline \multicolumn{7}{|l|}{ SURFnet } \\
\hline \multicolumn{7}{|l|}{ SWITCH } \\
\hline \multicolumn{7}{|l|}{ URAN } \\
\hline & Más & $75 \%$ & $25 \mathrm{a}$ & & Men & \\
\hline
\end{tabular}

Por su propia naturaleza, entre esos colectivos se encuentran en todas ellas las universidades y los organismos públicos de investigación.

Otro colectivo muy habitual es el de los departamentos de investigación de los hospitales.

Más allá de esos colectivos, las distintas redes académicas, por motivos históricos 0 políticos, dan adicionalmente servicio a otros grupos de usuarios públicos o sin ánimo de lucro, a menudo relacionados con los usuarios tradicionales: educación primaria y secundaria; sistemas sanitarios; bibliotecas, museos y otros organismos culturales; determinados organismos públicos; 0 , para proyectos específicos, departamentos de I+D+i de empresas. 


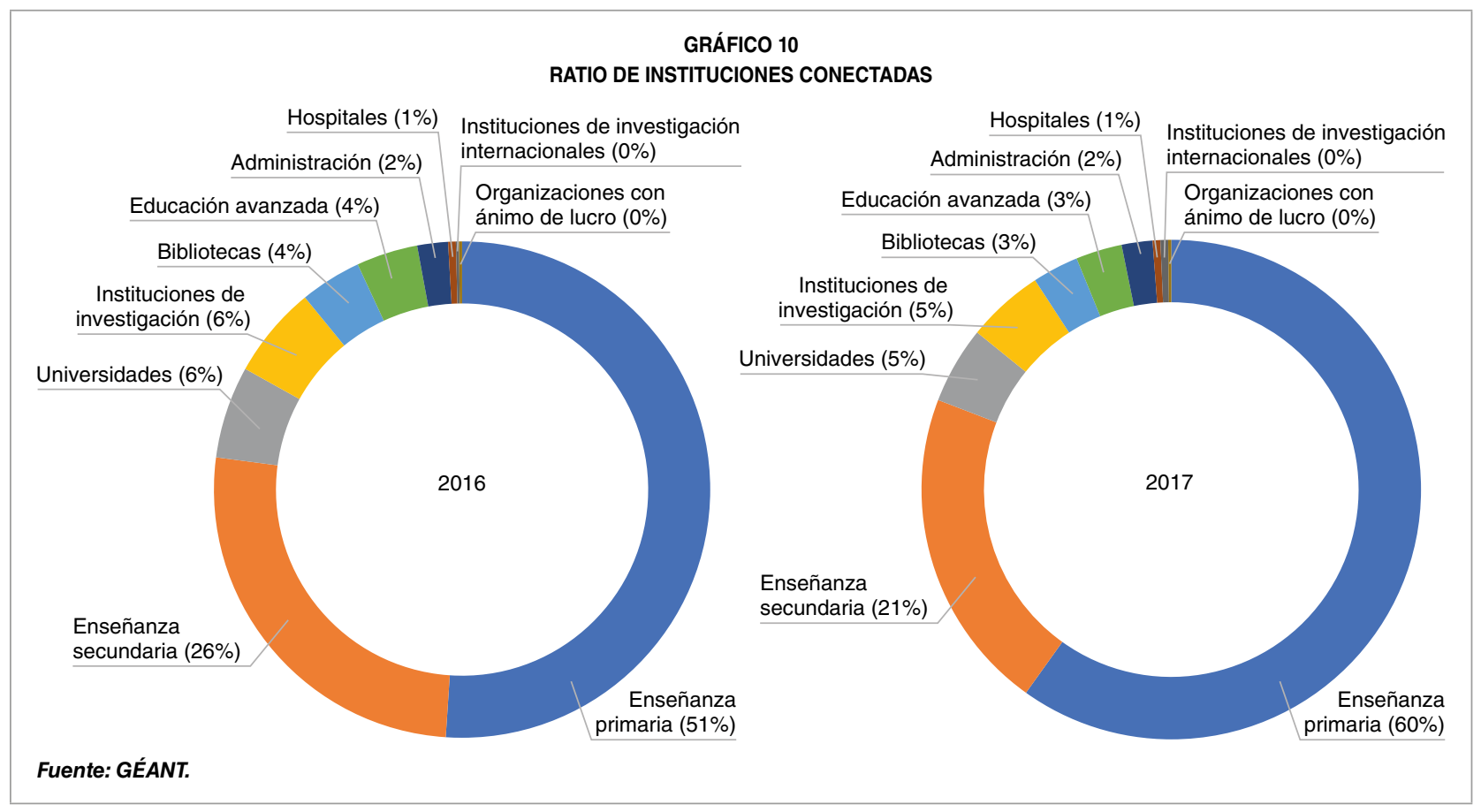

Con independencia de cuáles sean los grupos de usuarios aceptados por las redes académicas y de investigación, estas deben dotarse también de políticas de uso aceptable, que impidan la comercialización (reventa) de los servicios de conectividad, que fijen la política de acceso y de seguridad a cumplir por las instituciones afiliadas y que establezcan pautas en materia de protección de datos o de respeto a derechos de terceros (incluyendo derechos de propiedad intelectual), etcétera.

\section{RedIRIS como red académica y de investigación española}

\subsection{RedIRIS: función, organización y servicios}

RedIRIS (https://www.rediris.es/) es la Red Nacional para la Investigación y la Educación (National Research and Education Network, NREN) española, y ofrece servicios de comunicaciones avanzadas a más de quinientas instituciones de la comunidad científica y académica (sobre todo universidades, centros científicos e infraestructuras científico, técnicas singulares o ICTS).

RedIRIS es una infraestructura científica TIC cuyo titular es el Ministerio de Ciencia e Innovación (MINCI), que ha delegado las competencias relativas a RedIRIS a favor de Red.es MP, entidad pública empresarial dependiente del Ministerio de Asuntos Económicos y Transformación Digital. Red.es, que lleva haciéndose cargo de la gestión de RedIRIS desde 2004, es la agencia pública encargada de promover la transformación digital en España.

RedIRIS se puso en marcha en el año 1988, con el objetivo de ofrecer a las universidades y centros científicos una red troncal de comunicaciones propia para poder transferir grandes cantidades de información de forma eficiente y segura, facilitando así la colaboración remota entre estos centros y su participación en proyectos nacionales e internacionales, en particular proyectos de e-ciencia, que requieren transferencias masivas de datos. 


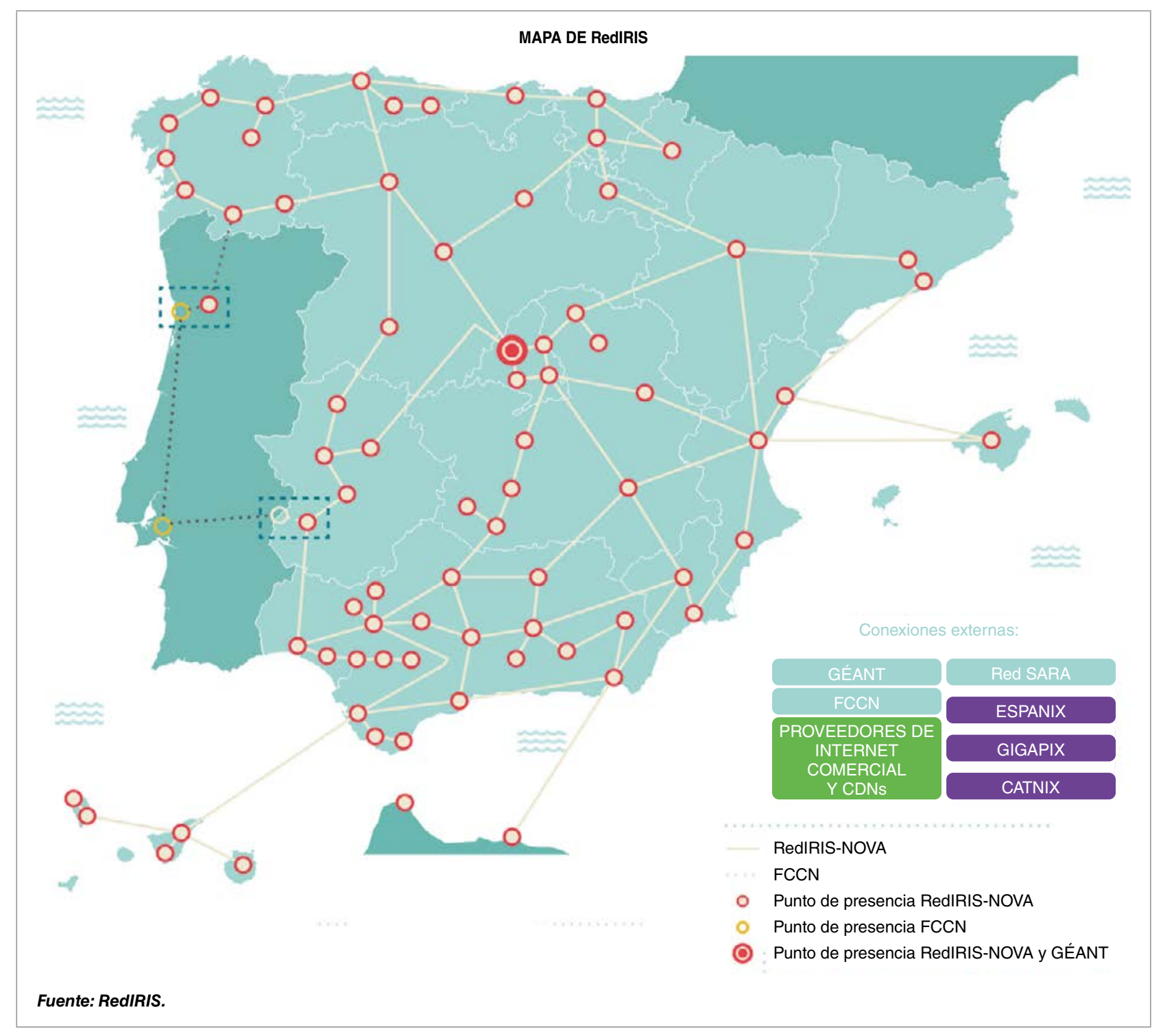

Para dar esos servicios de conectividad avanzada, RedIRIS gestiona en la actualidad una red troncal de unos 15.000 km de fibra óptica, (incluyendo más de 2.000 km de fibra submarina), con más de setenta puntos de presencia repartidos en todas las comunidades autónomas.

RedIRIS es una moderna infraestructura tecnológica, cuyo coste se eleva a más de 100 millones de euros (financiados en buena parte con fondos FEDER), y que se contrató usando fórmulas innovadoras. (A través de un diálogo competitivo se adquirieron IRUS, derechos de uso de muy larga duración sobre pares de fibra). Este modelo basado en la gestión de fibra óptica ofrece importantes avances tecnológicos en capacidad (casi ilimitada) y flexibilidad, lo que facilita la colaboración nacional e internacional. Gracias a esa potente red troncal, RedIRIS puede ofrecer a sus instituciones afiliadas múltiples canales de 10 gbps (10.000 Mbps), que pasarán a ser canales de $100 \mathrm{Gbps}$ cuando concluya la renovación del equipamiento óptico actualmente en ejecución.

RedIRIS presta sus servicios en estrecha colaboración con otras redes académicas y $\triangleright$ 
científicas, tanto autonómicas como internacionales. Entre estas últimas destaca la red académica paneuropea Géant, en cuya gestión participa RedIRIS, y a través de la cual se conecta tanto con las Redes Académicas y de Investigación Nacionales (NREN) de los demás países europeos, como con redes de investigación de otros continentes: Internet2 (EE UU), RedCLARA (América Latina), EUMEDCONNECT (Norte de África), TEIN (Asia-Pacífico), etcétera.

A través de RedIRIS se interconectan sedes de universidades y centros de investigación; se cubren las necesidades de conectividad avanzada de proyectos de investigación como LHC (Gran Colisionador de Hadrones) del CERN, ELIXIR (ciencias de la vida) o VLBI (radioastronomía); y se facilita el acceso a instrumentos y recursos científicos como los telescopios de Canarias, la Reserva de la Biosfera de Doñana, la Red Española de Supercomputación, etcétera ${ }^{8}$.

RedIRIS presta, además, otros servicios TIC a la comunidad académica y científica, en los ámbitos de seguridad (gestión de incidentes de seguridad, mitigación de ataques de denegación de servicio, filtrado antispam, certificados digitales), identidad digital (SIR), movilidad en wifis académicas (eduroam), herramientas colaborativas, transferencia de ficheros de gran tamaño, contratación colectiva de servicios cloud, soporte a ciertos servicios de administración electrónica, asesoramiento y difusión (incluyendo eventos y cursos de formación).

El éxito del modelo de las NREN en general, $y$ de RedIRIS en particular, ha llevado a que hayan solicitado la colaboración de RedIRIS

8 Ver fichas con ejemplos de casos de uso de RedIRIS en http://www. rediris.es/difusion/publicaciones/casos_uso/index.html.es

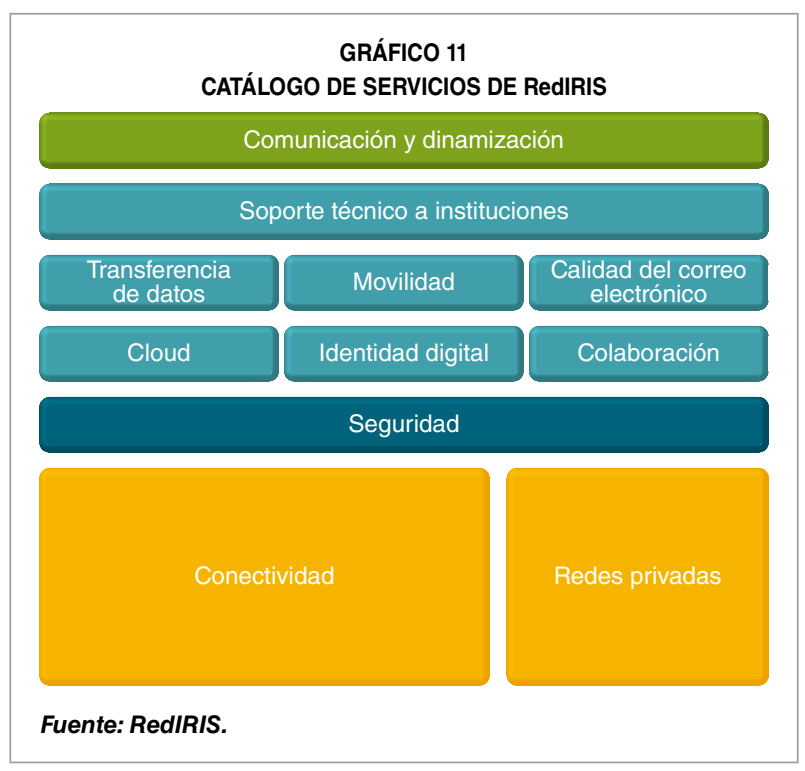

tanto redes académicas y científicas autonómicas como de otros países, e incluso otras redes dentro de la Administración que buscan replicar las ventajas de RedIRIS en términos de servicio y coste (por ejemplo, la red militar nacional).

\subsection{RedIRIS como infraestructura científica y técnica singular}

RedIRIS se encuentra incluida en el Mapa de Infraestructuras Científicas y Técnicas Singulares (ICTS) del Ministerio de Ciencia e Innovación (MINCl). Este mapa agrupa a grandes infraestructuras científicas del país, que, por su elevado coste de inversión y operación, y por su importancia y carácter estratégico, se ponen a disposición de la comunidad científica. Entre las ICTS hay observatorios astronómicos, supercomputadores, grandes laboratorios, plataformas, sistemas de observación científica...

El MINCI revisa periódicamente las infraestructuras que están en ese mapa, y para ello solicita a todas las que quieran figurar en el mismo que le remitan un plan estratégico, que es luego evaluado por un panel de expertos $\triangleright$ 
internacionales, el CAIS (Comité Asesor de Infraestructuras Singulares).

En 2017, el MINCI solicitó a las ICTS que elaborasen un Plan Estratégico 2020, cuya evaluación concluyó a finales de 2018. Ese plan incluye un análisis de la infraestructura y su entorno, la formulación de una misión y una visión, la definición de líneas estratégicas (plasmadas en objetivos y acciones concretas, con estimación de costes y plazos) y un plan de inversiones.

El Plan Estratégico 2020 de RedIRIS como ICTS define:

\section{La misión de RedIRIS}

Proporcionar una excelente conectividad, y otros servicios TIC complementarios, a las instituciones académicas y científicas españolas, que en estrecha colaboración mutua y con las redes académicas y de investigación autonómicas e internacionales facilitan la colaboración remota y el acceso a e-infraestructuras a escala nacional e internacional.

\section{La visión de RedIRIS}

Ser el socio de confianza de las universidades y centros científicos españoles en el ámbito de las TIC, promoviendo la cooperación entre dichas instituciones y ofreciéndoles un catálogo de servicios TIC, incluyendo conectividad de alta capacidad y otros servicios complementarios, gestionados o contratados de forma centralizada, que impulsen la e-ciencia y el aprendizaje avanzado.

El Plan Estratégico 2020 se articula en torno a cuatro ejes:

1. Consolidación de la infraestructura, lo cual incluye, en particular:
- La renovación del equipamiento óptico a través del diálogo competitivo que se está llevando a cabo, por un importe de hasta 23 millones de euros.

- La renovación de equipamiento IP y de seguridad.

- Extensiones de la red de fibra óptica a más centros académicos y científicos, en colaboración con estos.

2. Mejora de la cartera de servicios de RedIRIS (más seguridad, fiabilidad y valor añadido en los servicios existentes, y puesta en marcha de nuevos servicios).

3. Poner en el centro del modelo de gestión a las instituciones afiliadas a RedIRIS (user-centric approach), reforzando la relación con nuestros principales colaboradores (redes académicas internacionales y autonómicas, grupo TIC de la CRUE, ICTS, Red Española de Supercomputación, e INCIBE y CCN/CNI en materia de ciberseguridad).

4. Mejora del posicionamiento en e-ciencia, ofreciendo servicios que ayuden a hacer realidad el modelo de ciencia abierta en la nube (con foco específico en transferencia de datos; seguridad, identidad digital y servicios compartidos, sobre todo cloud). También se prevé una mayor coordinación con la otra ICTS de e-ciencia, la Red Española de Supercomputación.

Esos cuatro ejes estratégicos se concretan en un ambicioso listado de 12 objetivos y 16 acciones concretas, y en un Plan de Inversiones.

EI CAIS evaluó de forma muy positiva (excellent) todos los principales apartados del plan (carácter estratégico y singular, acceso, producción y rendimiento, y el plan estratégico en sí). También validó todas las inversiones $\triangleright$ 


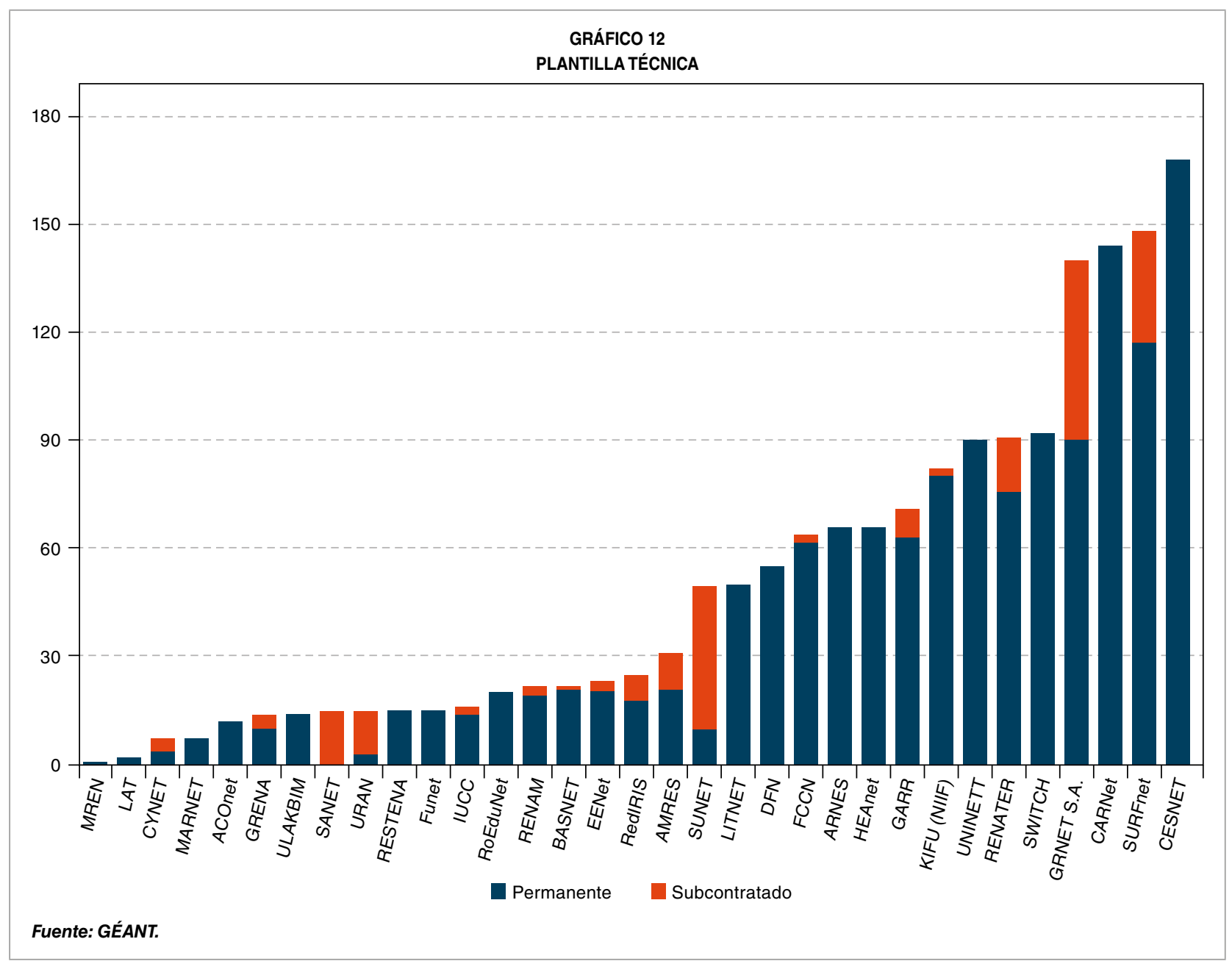

propuestas, a expensas de que luego se consiga o no la asignación de suficientes fondos FEDER, y de financiación para la parte nacional (en particular, cofinanciación para las extensiones de fibra por parte de los organismos afectados).

La principal preocupación expresada por el CAIS es que RedIRIS no disponga de suficientes medios humanos para poder desarrollar su plan estratégico, ya que su dotación de personal es muy reducida si se compara con otras

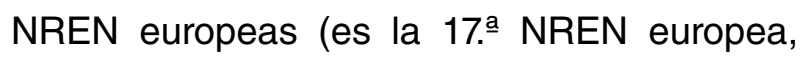
pese a lo cual dispone de un catálogo de servicios similar al de otras NREN con más recursos, y su actividad es valorada en términos positivos por sus usuarios).

\section{Conclusiones}

En un modelo basado en la economía del conocimiento, los Estados más avanzados invierten en educación y en I+D+i, y parte de esa inversión va destinada a garantizar que estos sectores estratégicos dispongan de las infraestructuras TIC adecuadas para llevar a cabo su tarea. En ese contexto se encuadra la inversión en redes académicas y científicas nacionales (NREN), como organismos encargados de garantizar a esos colectivos servicios de conectividad avanzada, tanto a escala nacional como a escala internacional, a través de la colaboración con sus homólogos de otros países, con los que configuran una Intranet $D$ 
Global de la Investigación. Las NREN también cubren otros servicios TIC muy relevantes, como los de seguridad y de identidad digital, y fomentan el trabajo colaborativo y el acceso a recursos compartidos, como los servicios cloud.

RedIRIS, como NREN española, cubre desde 1988 esa necesidad a escala nacional, en estrecha colaboración con las redes académicas y científicas autonómicas e internacionales, y con las más de quinientas instituciones afiliadas. El modelo empleado permite ofrecer servicios de muy alta capacidad y muy flexibles, obteniendo grandes ahorros, lo que ha llevado a que otros organismos de la Administración se estén inspirando en él para realizar despliegues similares.

\section{Bibliografía}

Davies, H., \& Bressan, B. (eds.) (2010). A history of international research networking: The people who made it happen. John Wiley \& Sons.

Comisión Europea (2016). Communication from the Commission to the European Parliament, the Council, the European Economic and Social Committee and the Committee of the Regions: European Cloud Initiative - Building a competitive data and knowledge economy in Europe (COM (2016) 178 final).

The World's Most Valuable Resource is no longer Oil but Data. (May 6th, 2017). The Economist. https://www.economist.com/leaders/2017/05/ 06/the-worlds-most-valuable-resource-isno-longer-oil-but-data

Vèa, A. (2013). Cómo creamos Internet. Península. 